\title{
COMMISSION 8: ASTROMETRY
}

\author{
(ASTROMÉTRIE)
}

\author{
PRESIDENT: Wen-Jing Jin \\ VICE-PRESIDENT: Imants Platais \\ ORGANIZING COMMITTEE: E. Costa, C. Ducourant, I.I. Kumkova, M.G. \\ Lattanzi, C.-L. Lu, S. Roeser, E. Schilbach, H. Schwan, R.C. Stone
}

\section{BUSINESS SESSION (Chaired by I. Platais}

The business meeting was opened by the Vice-President, Imants Platais, in lieu of the President, Wenjing Jin, who could not attend the General Assembly (GA). This was the first term for the Commission under the new name after the merger at the Manchester GA of the former Commissions 8 and 24. During the past triennium there was limited opportunity to meet except at the Journées "Systèmes de Référence Spatio-temporels" annual meetings and the JENAM 2001. This GA at Darling Harbour Convention Center in Sydney catered for a variety of 'astrometry-flavored' meetings to further promote our science and collaborations.

The vice-president presented the agenda which was unanimously approved. The business meeting was attended by 30 participants. The meeting approved N. Zacharias as secretary of minutes and R. Gaume with D. Pourbaix as canvassers.

A minute of silence was observed in memory of the Commission members who passed away since the Manchester GA: Christian de Vegt, Olexander Molotaj, Lai Wan, and a good friend of many Commission members - Mrs Jeannine Kovalevsky.

\subsection{Commission Activities in 2000-2003}

Platais informed the meeting that the EC has not supported the proposed Joint Discussion for this GA. Thus the last international astrometry meeting was IAU Colloquium 180 "Towards Models and Constants for Sub-Microarcsecond astrometry" held at the U.S. Naval Observatory in March 2000. The Commission has supported two unsuccessful meeting proposals and two accepted meetings - both at this GA (JD 16 and SPS 3). A letter of support was written for the GAIA mission, which continues to be on track at the European Space Agency.

Information dissemination is largely conducted via the Commission's WWW homepage at http://center.shao.ac.cn/IAU_COM8. A new homepage will be installed by the end of 2003 at the Johns Hopkins University's (USA) website. A total of six newsletters and two issues of "letters to members", as well as various draft resolutions were circulated during the 2000-2003 period. In this electronic age regular mail in the Commission's business has essentially ceased to exist, hence it is of utmost importance to update the list of individual e-mail addresses. All members are strongly encouraged to check their personal data in the IAU membership directory and report the changes to the IAU Secretariat.

Platais thanked the Organizing Committee for the excellent job in preparing for the GA and many individuals for their contribution to the triennium report. 


\subsection{New Commission Members and Consultants}

The following new Commission members were confirmed:

a) IAU members requesting membership of Commission 8

Jean-Eudes Arlot (Institut MC\&CE, France)

Arthur Bradley (Spacecraft System ES, USA)

Nicole Capitaine (Observatoire de Paris, France)

Dimitri Pourbaix (Institut A\&A, Belgium)

William Thuillot (Institut MC\&CE, France)

b) New IAU members requesting membership of Commission 8

Marcelo Assafin (Observatorio do Valongo/UFRJ, Brazil)

Jos De Bruijne (ESA-ESTEC, The Netherlands)

Theo ten Brummelaar (CHARA, USA)

Yuri Kolesnik (Institute of Astronomy RAS, Russia)

Vera Martin (Universidade Estadual de Feira de Santana, Brazil)

Paulo Poppe (Universidade Estadual de Feira de Santana, Brazil)

Yuri Protsyuk (Nikolaev Observatory, Ukraine)

Damien Ségransan (Observatoire de Genève, Switzerland)

Alexander Shulga (Nikolaev Observatory, Ukraine)

Richard Smart (Osservatorio di Torino, Italy)

Drago Spoljaric (University of Zagreb, Croatia)

Zhenghong Tang (Shanghai Astronomical Observatory, China PR)

c) New Commission consultants

Nigel Hambly (University of Edinburgh, UK)

This brings the total membership to 226 (including 3 consultants), representing 31 countries although USA, France, China PR, Germany, and Russia combined contribute more than half of the active members.

\subsection{Election Results, New Commission Officers}

Following the long-standing IAU tradition, the vice-president for the 2000-2003 triennnium - Imants Platais - was nominated to serve as the Commission's next president. Considering the geographical factor in the succession of the Commission's leadership, only one member of the OC - Irina Kumkova - was nominated and received unanimous support. Following the IAU EC recommendations and the discussions at the at the Manchester GA, the OC 2003-2006 of Commission was down-sized from a previous total of 11 to 10 . Thus there were only three vacancies for the $\mathrm{OC}$ to be filled in. Out of eight candidates nominated for the OC, the following new OC members were selected in a two-stage voting process: D.W. Evans, R.-D. Scholz, and M. Soma.

The new Commission officers for 2003-2006: 
Imants Platais (USA) - president

Irina Kumkova (Russia) - vice-president

Organizing Committee: Edgardo Costa (Chile), Christine Ducourant (France), Dafydd Evans (UK), Wen-Jing Jin (China PR) - ex officio, Mario Lattanzi (Italy), Chunlin Lu (China PR), Ralf-Dieter Scholz (Germany), Mitsuru Soma (Japan)

This list of Commission's officers was approved by acclamation.

\subsection{Report of the $\mathrm{AC} / \mathrm{CdC}$ Working Group}

Urban (USNO) presented a report on the Astrographic Catalogue/Carte du Ciel working group, prepared by both him and A. Fresneau - the current chairman of the WG who could not attend the GA. A brief overview of the $\mathrm{AC} / \mathrm{CdC}$ project was presented for those unfamiliar with it. He then explained that due to the inherent accuracies of the positions that could be obtained ( 200 mas) and the large epoch span (upwards to 100 years), proper motions at the 2 mas $\mathrm{yr}^{-1}$ level could be expected when combined with modern astrometric observations. This was indeed performed using the published version of the Astrographic Catalogue and combining the resulting positions with those from the Tycho UCAC projects to produce the proper motions. Since few of the existing $\sim 7500 \mathrm{CdC}$ plates have ever been measured, a measurement would yield proper motions better than from the currently available data.

Urban reviewed work performed within the WG. Specifically, the Automatic Plate Measuring machine (APM, Cambridge) was used by R. Argyle to measure the 500 Bordeaux zone $\mathrm{CdC}$ plates $\left(+11^{\circ}\right.$ to $\left.+17^{\circ}\right)$. These will be utilized to derive proper motions for the M2000 catalog. Argyle used the same machine to measure various Sydney zone CdC plates $\left(-65^{\circ}\right.$ to $\left.-51^{\circ}\right)$, provided by A. Vaughn. The San Fernando Observatory has also measured $\sim 400$ plates from their zone $\left(-10^{\circ}\right.$ to $\left.-2^{\circ}\right)$ and the data are now available. Observatories in Toulouse, Cordoba, and Uccle are investigating machines to measure the $\mathrm{CdC}$ plates. The results from these observatories is that the position errors are good globally to 150 to 300 mas, with some photometric information to within 0.35 mag.

Urban then raised the question of continuation of the WG. Fresneau, who has served as chairman for nine years, would like to hand over the leadership to someone else. After discussion, it was decided by the Commission to re-instate the WG with B. Bucciarelli (Italy) as the new chairwoman.

\subsection{General Discussion}

Hartkopf (USA) announced Special Session 3, "A New Classification Scheme for Double Stars", being held during the GA. Improved observational techniques have led to increasing overlap among previously isolated binary star period/separation regimes; one consequence is that different component naming schemes used by practitioners of these various techniques are beginning to cause confusion in component identifications. A new nomenclature scheme, based on the one used in the Washington Double Star catalog, has been developed in order to address this confusion. A catalog of all types of binaries (including stars with both stellar and sub-stellar companions) is being compiled as a part of this effort. Following a Type-C resolution adopted in Manchester (and supported by Commission 8), a sample portion of this catalog was prepared in time for discussion at the Sydney GA's Special Session. This Commission's support was requested (and subsequently obtained) for a second Type-C resolution to extend this catalog to the entire sky. 
Urban announced the availability of the 2nd data release of the USNO CCD Astrograph Catalog (UCAC2). In total 48 million stars over $86 \%$ of the sky are measured down to a red limiting magnitude equal to 16 . The catalog includes precise positions ( $\sigma_{\text {pos }} \sim 20$ mas), proper motions (from $\sigma_{\mu} \sim 1-2$ mas yr $^{-1}$ to $4-7$ mas $\mathrm{yr}^{-1}$ for the fainter stars), and the 2MASS $J H K$ photometry, all available on three CDs. Requests are encouraged to be sent to Norbert Zacharias (E-mail: nz@usno.navy .mil).

Griffin stressed the importance of increasing the attendance at the GA by young astronomers. This issue should be raised at all levels of the IAU hierarchy.

Seidelmann initiated a discussion on the need of an astrometry meeting. In the post-Hipparcos era it is not easy to convince the EC on the necessity of sponsoring an astrometric meeting. It may require some institution to take on the burden of organizing such a meeting.

van Altena and Johnston suggested to return back to the proven format of the former Commission $8 \& 24$ business meetings, i.e., reinstating the observatory reports.

\section{SCIENTIFIC SESSION (Chaired by K. Seidelmann)}

A talk by van Altena (Yale U.) in collaboration with T. Girard, D. Dinescu, I. Platais, C. Lopez, and D. Monet "The Yale/San Juan Southern Proper Motion Catalog 3" was an update on the current status of the SPM program. Absolute proper motions, positions, and photographic $B, V$ photometry have been obtained for $\sim 11$ million objects, down to $V=17.5$. The Catalog covers an irregular area of 3700 square degrees, between the declinations of $-20^{\circ}$ and $-45^{\circ}$, but excluding the Galactic plane. All observations were made with the 50-cm double astrograph of Cesco Observatory in El Leoncito, Argentina. The Catalog is based on full-plate scans using the USNO Precision Measuring Machine (PMM) and its image detection and centering software. The proper-motion precision,

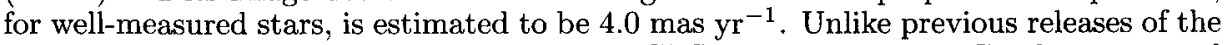
SPM Catalog, the proper motions are on the ICRS via the Hipparcos Catalog stars, and have an estimated systematic uncertainty of 0.4 mas $\mathrm{yr}^{-1}$. The recently begun SPM CCD survey program was also described. These observations will provide second-epoch astrometry, as well as improved photometry, for the remainder of the original Southern Proper Motion survey.

Lindegren (Lund Obs.) in collaboration with D. Dravins presented a talk "The fundamental definition of radial velocity". Radial velocity is an omnipresent concept in astronomy, yet no agreed, unambiguous definition existed before Resolutions C1 and C2 were adopted by the IAU 24th GA in Manchester (2000). A thorough examination of the concept, aiming at accuracy levels down to $1 \mathrm{~m} \mathrm{~s}^{-1}$, has been presented in Lindegren \& Dravins (2003). For measurement accuracies of order $1 \mathrm{~km} \mathrm{~s}^{-1}$ the traditional concepts are usually adequate, but for higher accuracies, second-order effects (in $v / c$ ) become significant even in a classical treatment (e.g. treatment of light-time effects and the conversion from redshift, $z$, to velocity). Further confusion is caused by relativistic effects, motions in the stellar atmospheres, etc. The proposed solution is to distinguish clearly between the geometrical concept of stellar radial motion, which we call "astrometric radial velocity", and the spectroscopically measured quantity, which we call (barycentric) "radial-velocity measure". Both concepts are precisely defined by the resolutions. To first order both agree with traditional concepts, but for present and future high-accuracy measurements, differences occur that may contain significant astrophysical information.

Argyle (Cambridge, UK) gave new insights on the Southern Coalsack "Collisional dynamics of stellar systems in the Southern Coalsack". The visual interstellar extinction 
in the Southern Coalsack is estimated from a photographic survey of proper motions and $\mathrm{B}, \mathrm{V}$ photometry of 60,000 stars as faint as apparent magnitude 14 over a field of view of $6^{\circ} \times 6^{\circ}$ (Fresneau et al, 2003). Selecting the stars with proper motions greater than 9 mas $\mathrm{yr}^{-1}$, produces a catalogue of 20,000 entries. To select the dwarf stars needed for this study, the work of Binney et al. (2000) is applied in which they correlate the B-V color with tangential velocities. This leads on to kinematic and photometric distances assuming that the star is on the main sequence. The total extinction $A_{v}$ is derived as a function of kinematic distance for 20,000 stars in the Southern Coalsack region, a magnitude excess of at least 0.80 equivalent to a distance of $180 \pm 60 \mathrm{pc}$ from the Sun. For the 1,000 stars at this distance galactic orbits are computed which imply a mass for the Southern Coalsack of $2500 \pm 1000 M_{\odot}$. Plates from the Sydney Astrographic project have been scanned on the APM at Cambridge to give much improved positions and proper motions but it is clear that the quality of the plate material is deteriorating and the remaining plate material should be digitized as soon as possible.

Arlot (Institut MC\&CE, Paris) highlighted the potential of photometric observations for the Jovian system "Observation campaigns PHEMU of natural planetary satellites and new results". The four Galilean satellites of Jupiter have their orbits in the same plane and mutual occultations and eclipses occur when the Earth and the Sun pass through this plane every 6 years. The photometric observation of these events allows the relative positions of these satellites to be obtained with a high accuracy. Advantage of the last opportunity of such a mutual occultation event was taken in 2003 by making the observations within the international network of small aperture telescopes (from 20 $\mathrm{cm}$ to $2 \mathrm{~m}$ ). More than 1200 observations have been made from 1973 to 2003 and these can be fit to the theoretical models in order to get ephemerides of high accuracy. The first results show that there is the possibility to detect an acceleration in the motion of Io and Europa, a signature of the energy dissipated by the tides inside these bodies.

Vaughan (Macquarie U.) reported on the status AC/CdC plates "The legacy of Sydney Observatory Astrographic Plates". The plates of Sydney Observatory (AC + CdC +2 nd epoch plates) and Melbourne Observatory $(\mathrm{AC}+\mathrm{CdC})$ are stored in the Library at Macquarie University in Sydney. The Melbourne plates $\left(-62^{\circ}\right.$ to $\left.-90^{\circ}\right)$ have not yet been re-examined, but the Sydney plates $\left(-58^{\circ}\right.$ to $\left.-62^{\circ}\right)$ are presently being cataloged and re-measured. The Sydney $\mathrm{CdC}$ plates have never before been measured. About 700 $\mathrm{AC}$ and $\mathrm{CdC}$ plated have been measured at APM to about $3 \mu \mathrm{m}$ accuracy $\left(0^{\prime \prime} 3\right)$. These include all plates with $|b|<4^{\circ}$ over a wide range of longitudes. The most useful science to arise so far has been proper motions to $m_{\mathrm{pg}}=14$ from $\mathrm{CdC}$ plates near the Southern Coalsack (Fresneau et al. 2003).

van Altena (Yale U.) in collaboration with D. Hoffleit gave a detailed account on the "Schlesinger's Telescope: A History of the Yale 26-inch Refractor in The Commonwealth". The Schlesinger 26-inch refractor was destroyed by a fire-storm on January 18,2003 , along with the other telescopes on Mount Stromlo, the workshop, library and many of the residences. Frank Schlesinger, began planning for the establishment of a southern observatory when he arrived at Yale in 1920. A large photographically corrected 26-inch objective was ground, polished and completed by James McDowell in 1923 , while the tube and mounting of the telescope was being built largely in the Yale shops in New Haven. Installation of the 26-inch refractor at the site near Johannesburg (South Africa) was completed in early June and dedicated by the Prince of Wales on June 22, 1925. The principal observational program for the 26-inch refractor was the determination of parallaxes of the bright stars and it was continued until 1952, when the telescope was moved to Mt. Stromlo, Australia, due to the deteriorating sky conditions 
in Johannesburg. The parallax program continued at Mt. Stromlo until 1963 when the telescope was donated to the Commonwealth Observatory. Approximately 2,000 stellar parallaxes were determined from the 70,000 plates taken with the refractor. The firestorm was a sad ending to a telescope that played a major role in defining our knowledge of the distances, motions and masses of the brighter stars during the middle of the 20th century.

Platais (Johns Hopkins U.) presented the status of the "WIYN Open Cluster Study (WOCS)". Among 1,500 known open clusters only ten can be considered to have been studied comprehensively. The WOCS has set out to enlarge the number of the so-called fundamental clusters by a factor of two combining the efforts by astrometric, photometric, and spectroscopic means. Platais is responsible for deep astrometry (positions and proper motions down to $V=21-23$ ) in a few selected open clusters in both hemispheres. The Kitt Peak Mayall 4-m, WIYN 3.5-m, and CTIO Blanco 4-m in combination with large-format $\mathrm{CCD}$ mosaic cameras are the primary instruments to achieve this goal. With the example of NGC 188 it has been shown that excellent proper motions $\left(\sigma_{\mu}=0.15\right.$ mas $\left.\mathrm{yr}^{-1}\right)$ can be derived (Platais et al. 2003) allowing one to reach convincing separation between the cluster and field stars. Such cluster fields are also a valuable source of precise positions ( $\sigma_{\text {pos }}=3$ mas) helping to extend the ICRS to much fainter magnitudes.

On behalf of I. Kanaev, A. Tikhonov, K. Naumov, V. Koupriyanov, O. Rusakov, M. Sidorov, and V. Frolov, Kumkova (Institute of Applied Astronomy, St. Petersburg) presented a paper "Observations of M67 and Other Open Clusters on Magis; Pilot Results". The results of relative proper motion determinations for the open clusters M67 and NGC 7243 with the Pulkovo meridian automatic horizontal instrument - MAGIS ( $D=190 \mathrm{~mm}, \mathrm{~F}=800 \mathrm{~cm}, 768 \times 580$ pix CCD) were presented. The discussion and comparison of the results obtained by MAGIS and the Normal Astrograph of Pulkovo observatory were provided. It was shown that the positional errors from observations with MAGIS in the same field but on different nights are small - only 3-20 mas. Proper motions of about 20 stars calculated from the MAGIS mean positions in combination with observations by the Normal Astrograph show good agreement with the data obtained by other authors. A pilot study indicates only some small differences between the mean positions of stars on the MAGIS system and and those on the system of a Normal Astrograph. Thus the observations by MAGIS can successfully continue the long series of the Pulkovo Normal Astrograph observations (over a 100-yr span) to derive precise proper motions.

I. Platais

Vice-President of the Commission

\section{References}

Binney, J, Dehnen, W. \& Bertelli, G. 2000, MNRAS, 230, 597

Fresneau, A, Vaughan, A. \& Argyle, R.W., 2003, AJ, 125, 1519

Lindegren, L. \& Dravins, D. 2003, A\&A, 401, 1185

Platais, I., Kozhurina-Platais, V., Mathieu, R.D, Girard, T.M. \& van Altena, W.F. 2003, AJ, in press (astro-ph/0309749) 